\title{
Angle Estimation Using Hahn Moments for Image Analysis
}

\author{
C. Camacho-Bello and J.J. Báez-Rojas \\ Instituto Nacional de Astrofísica, Óptica y Electrónica, \\ Luis Enrique Erro No. 1, 72840, Puebla, México
}

\begin{abstract}
We present a new approach for angle estimation in binary images from Hahn moments, which provide an approximate estimate with short computational times. The method proposed retrieving the angle formed from a reference point to another, through a multiple linear regression and a set of Hahn moments obtained in a training database. Finally, we discuss the performance analysis of our approach under noise conditions and scale change.
\end{abstract}

Keywords: angle estimation, discrete orthogonal moments, multiple linear regression, image analysis.

\section{Introduction}

Discrete orthogonal moments are widely used in pattern recognition, image analysis and machine vision, have the ability to characterize, evaluate, and manipulate visual information, with minimum redundant information. In 2001, Mukundan et al. 1] introduced the notion of discrete orthogonal moments, used a set of discrete Tchebichef polynomials as moment functions base. In this paper it was shown that implementation of discrete orthogonal moments does not involve any numerical approximation since the basis set is orthogonal in the discrete domain of the digital image. Similar to Tchebichef moments, Yap et al. [2] introduced the Krawtchouk moments based on the discrete Krawtchouck polynomials and has the ability to extract local features from any region of interest in an image unlike the Tchebichef moments, which extract global features. Recently, Yap et al. 3] proposed Hahn moments, which have the ability to extract local or global features of an image when changing the parameters, $\alpha$ and $\beta$ in Hahn polynomial. The variation of these parameters can form various types of discrete orthogonal moment: Tchebichef moments $(\alpha=\beta=0)$ and Krawtchouk moments $(\alpha=(1-p) t, \beta=p t$ and let $t \rightarrow \infty)$. This makes Hahn moments a useful set of image feature descriptors [4].

Numerous works on orthogonal moments 1223 only focus on the extraction of global and local features, and not on information related to the shape of the image. Moreover, $\mathrm{Hu}$ [5] proposed the central moments, which can find geometrical patterns such as density, centroid, size and orientation of the image. The orientation angle is obtained by the method of principal axes, which is limited to $|\theta|<45^{\circ}$ and patterns that are not circularly symmetric.

E. Bayro-Corrochano and E. Hancock (Eds.): CIARP 2014, LNCS 8827, pp. 127-134 2014.

(C) Springer International Publishing Switzerland 2014 
In this paper addresses the problem of estimating the angle formed from a reference point to another regardless of the symmetry type . To achieve this, we propose predicting the angle in the image using information provided by discrete orthogonal moments and a set of training images. Several experiments were performed to validate the effectiveness of proposed method in terms of scale change and under noise condition. In addition, we compared the orientation using the central moments for a set of images that do not have circularly symmetric.

\section{Hahn Moments}

In general, Hahn moments of order $(n+m)$ of a two-dimensional image with intensity function $f(x, y)$, are defined [3] as

$$
H_{n, m}=\sum_{x=0}^{M-1} \sum_{y=0}^{N-1} \widetilde{h}_{n}^{\left(\alpha_{1}, \beta_{1}\right)}(x ; N) \widetilde{h}_{m}^{\left(\alpha_{2}, \beta_{2}\right)}(y ; M) f(x, y),
$$

where $\widetilde{h}_{n}^{\left(\alpha_{1}, \beta_{1}\right)}$ are weighted Hahn polynomials and $\alpha_{1}, \beta_{1}, \alpha_{2}, \beta_{2}$ are the control parameters to global or local feature extraction of a digital image, which are given as follows

$$
\begin{aligned}
& \alpha_{1}=\frac{x_{c}}{N} t_{1}, \beta_{1}=\left(1-\frac{x_{c}}{N}\right) t_{1}, \\
& \alpha_{2}=\frac{y_{c}}{M} t_{2}, \beta_{2}=\left(1-\frac{y_{c}}{M}\right) t_{2}
\end{aligned}
$$

where $\left(x_{c}, y_{c}\right)$ are the central points of the region of interest to local feature extraction, and factors $t_{1}$ and $t_{2}$ indicate if the moments are local or global, when values of $t$ increase the moments become more local, and otherwise, when closer to zero are more global. Moreover, the calculation of orthogonal polynomials cause numerical instability for higher orders. To reduce this problem propose using recurrence relations with respect to $x$ [6] [7, unfortunately also causes numerical instability for images with $N$ large [8]. In this paper, we only use low-order polynomials for the calculation of the orthogonal moments, which provide global information of the image to be analyzed. In order to improve the calculation efficiency of Hahn polynomials we propose a recurrence relation with respect to $n$, which has smaller number of operations that some of the proposals of recurrence relations [910. The recurrence relation for Hahn polynomials is given by

$$
\begin{aligned}
A_{n}^{(\alpha, \beta)} \widetilde{h}_{n}^{(\alpha, \beta)}(x ; N)= & \widetilde{h}_{n-1}^{(\alpha, \beta)}(x ; N)\left(x-B_{n}^{(\alpha, \beta)}\right) \sqrt{(\alpha+\beta+2 n)^{2}-1} \\
& -\widetilde{h}_{n-2}^{(\alpha, \beta)}(x ; N) A_{n-1}^{(\alpha, \beta)} \sqrt{\frac{\alpha+\beta+2 n+1}{\alpha+\beta+2 n-3}}
\end{aligned}
$$


where

$$
\begin{aligned}
& A_{n}^{(\alpha, \beta)}=\frac{\sqrt{n(\alpha+\beta+n)(\alpha+n)(\beta+n)(N-n)(\alpha+\beta+n+N)}}{\alpha+\beta+2 n}, \\
& B_{n}^{(\alpha, \beta)}=\frac{\alpha-\beta+2 N-2}{4}+\frac{\left(\beta^{2}-\alpha^{2}\right)(\alpha+\beta+2 N)}{4(\alpha+\beta+2 n-2)(\alpha+\beta+2 n)} .
\end{aligned}
$$

For the initial numerical calculation, the normalized Hahn polynomials of zero and first orders can be calculated as follows,

$$
\begin{aligned}
\widetilde{h}_{0}^{(\alpha, \beta)}(x ; N)= & \widetilde{h}_{0}^{(\alpha, \beta)}(x-1 ; N) \sqrt{\frac{(N-x)(b+x)}{(N+\alpha-x) x}}, \\
\text { with } \widetilde{h}_{0}^{(\alpha, \beta)}(0 ; N)= & \sqrt{(\alpha+1)_{b}(\alpha+\beta+1) /(N+\alpha)_{b+1}}, \\
\widetilde{h}_{1}^{(\alpha, \beta)}(x ; N)= & {[(\alpha+\beta+2) x-(\beta+1)(N-1)] \sqrt{\frac{\alpha+\beta+3}{(\alpha+1)(\beta+1)}} } \\
& \times \sqrt{\frac{1}{(N-1)(N+\alpha+\beta+1)}} \widetilde{h}_{0}^{(\alpha, \beta)}(x ; N)
\end{aligned}
$$

where $(\cdot)_{k}$ is the symbol of Pochhammer. Note that the initial values for the recurrence relation are free from factorials terms and gamma functions.

\section{Proposed Method}

Our goal is to build a function that take a matrix moment $H_{n, m}$ as input and predict the angle $\theta$ related to the shape of the image, this can be achieved by adopting a machine learning approach in which a set of $I$ orthogonal moments $\left\{H_{n, m}^{1}, H_{n, m}^{2}, \ldots, H_{n, m}^{I}\right\}$ called training set are used to adjust the model parameters to a target vector $\theta_{i}$. The multiple linear regression model can relate to a linear combination of shape descriptors, which correspond to values of $\theta_{i}$, can be estimate as follows:

$$
\theta_{i}=\frac{1}{k} \sum_{n=0}^{L} \sum_{m=0}^{L} c_{n, m} H_{n, m}^{i},
$$

where $L$ is the maximum number of moments, $c$ are the parameters of the linear function, and $k$ is the scaling factor of the orthogonal moments [1]. In the literature, there are other methods to compute the scale invariance [412; these are constructed using a linear combination of geometric moment invariants. Unfortunately with the proposed transformation lose the orthogonality condition, which is necessary for this application. In order to evaluate the proposed method, we considered three different cases, as shown in Fig. 1.

In many cases, to determine the coefficients $c$ of Eq. 10 it is necessary to use a training set. Suppose that we have a training set $I$ observations, together with 


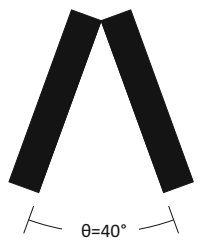

(a)

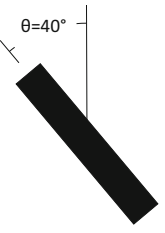

(b)

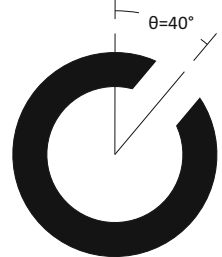

(c)

Fig. 1. Three different cases of images, which form angles from a reference point

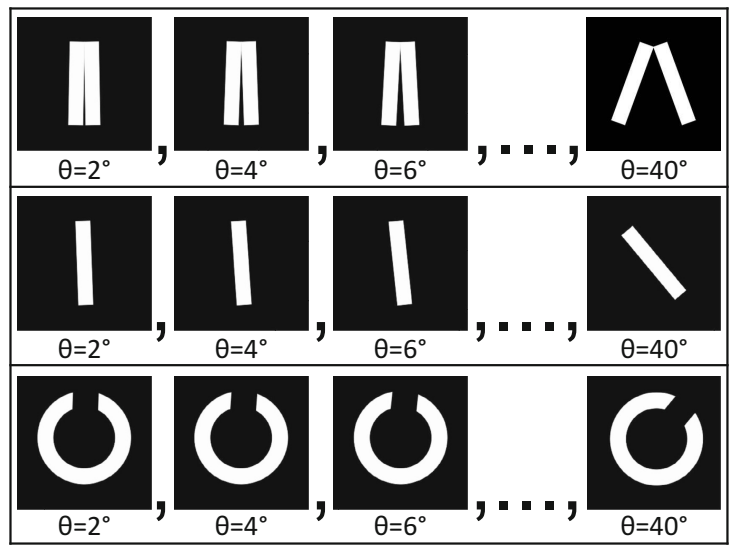

Fig. 2. Training set of the three different cases of Fig 1

the associated observations $\theta$ values. Figure 2 shows a set of training images comprised of $I=20$ images with angles of 2 to $40^{\circ}$, with intervals of $2^{\circ}$.

Note that to calculate Hahn moments for all the training images, it is needed to select the $\alpha_{1}, \beta_{1}, \alpha_{2}$, and $\beta_{2}$ parameters, which depend on the area where the angles changed either locally or globally. For example, in Fig. 1(a), the area of interest is located in the vertex of the two rectangles, using Eqs. (2) and (3), we can place the vertex in $\frac{x_{c}}{N}=\frac{1}{2}$ and $\frac{y_{c}}{M}=\frac{2}{3}$, and extracting local information with $t_{1}=t_{2}=60\left(\alpha_{1}=\beta_{1}=30, \alpha_{2}=40\right.$ and $\left.\beta_{2}=20\right)$. Moreover, in Figs. 11(b) and 1(c), the change of angles are located in whole image, therefore we use global features, such as those provided by the Tchebichef moments $\left(\alpha_{1}=\right.$ $\left.\beta_{1}=\alpha_{2}=\beta_{2}=0\right)$. With the $\alpha_{1}, \beta_{1}, \alpha_{2}$ and $\beta_{2}$ parameters selected, we can solve the problem of the estimate of angles using a least-squares approach, which minimizes the squared error function to find the values of the coefficients $c_{n, m}$. The solution of the corresponding values $c_{n, m}$ for the Fig. 1(a) is given by 


$$
\begin{aligned}
\theta_{1}^{*}= & 0.5767 H_{0,0}-0.5636 H_{0,1}-1.0265 H_{0,2} \\
& -0.2641 H_{0,4}+0.0403 H_{0,6}+0.4483 H_{0,7} \\
& -0.2326 H_{0,8}+0.2345 H_{2,0}-0.0932 H_{2,1} \\
& 0.1618 H_{2,6}+0.2225 H_{2,8}+0.2381 H_{4,2} \\
& +0.3040 H_{4,3}+0.8273 H_{4,5}-0.4319 H_{4,8} \\
& +1.0218 H_{6,0}+0.4706 H_{6,3}+0.0103 H_{6,7} \\
& +0.0103 H_{6,7}-0.2516 H_{6,9},
\end{aligned}
$$

Fig. 1(b) for the estimation of the angle is as follows

$$
\begin{aligned}
\theta_{2}^{*}= & 1.3900 H_{0,0}-0.5651 H_{0,18}-1.2632 H_{0,48} \\
& -1.9866 H_{8,0}+0.0411 H_{11,3}+1.2893 H_{17,1} \\
& +0.2391 H_{29,35}-0.1848 H_{32,22}-0.8620 H_{33,27} \\
& +0.4813 H_{34,10}+0.3600 H_{36,14}-0.4927 H_{40,0} \\
& -0.0904 H_{41,1}+0.4956 H_{42,50}-0.0568 H_{45,49} \\
& +0.9875 H_{46,48}+0.5910 H_{49,43}+0.0240 H_{50,22} \\
& +0.0240 H_{50,22}-0.0290 H_{50,30},
\end{aligned}
$$

and finally the estimation of Fig. 1(c) is given by

$$
\begin{aligned}
\theta_{3}^{*}= & 0.4387 H_{0,0}+1.6228 H_{6,1}-0.4413 H_{7,1} \\
& -0.1010 H_{13,0}-1.3183 H_{22,1}+0.6907 H_{23,1} \\
& -0.5600 H_{30,0}+2.9989 H_{32,17}+0.5492 H_{33,17} \\
& +0.2105 H_{38,30}-0.8196 H_{40,1}-0.4076 H_{41,1} \\
& +1.7151 H_{43,27}-0.3864 H_{44,50}-0.1056 H_{46,49} \\
& +0.6731 H_{48,22}-1.0699 H_{49,18}+0.8843 H_{49,40} \\
& +0.8843 H_{49,40}+0.0158 H_{49,48} .
\end{aligned}
$$

\section{Experiments}

To evaluate the fit of Eqs. (11), (12) and (13), we use 4 sets of test images; degradation-free images, degraded images with noise, images with scale change and a combination of both, which are described as follows

Set 1: free images of degradation. The test set consists of 40 free images of degradation, with size of $256 \times 256$ pixels, with angles of $1^{\circ}-39^{\circ}$, with intervals of $2^{\circ}$.

Set 2 : images degraded with noise. The test set consists of 40 images degraded with salt and pepper noise with a noise density of $0.02 \%$, with size of $256 \times 256$ pixels, with angles of $1^{\circ}-39^{\circ}$, with intervals of $2^{\circ}$. 
Set 3 : images with scale change. The test set consists of 40 images with scale change of $k=0.5$, with size of $128 \times 128$ pixels, with angles of $1^{\circ}-39^{\circ}$, with intervals of $2^{\circ}$.

Set 4: images degraded with noise and with scale change. The set consists of 40 images degraded with salt and pepper noise with a noise density of $0.02 \%$ and scale change of $k=0.5$, with size of $128 \times 128$ pixels, with angles of $1^{\circ}-39^{\circ}$, with intervals of $2^{\circ}$.

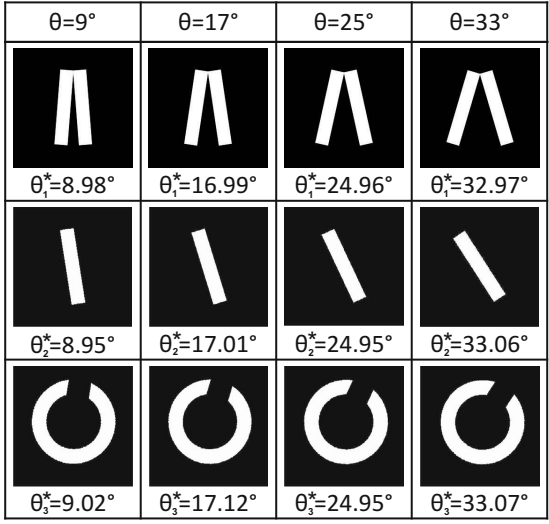

(a)

\begin{tabular}{|c|c|c|c|}
\hline$\theta=9^{\circ}$ & $\theta=17^{\circ}$ & $\theta=25^{\circ}$ & $\theta=33^{\circ}$ \\
\hline$\Lambda$ & $\Lambda$ & $\Lambda$ & $\Lambda$ \\
$\theta_{1}^{*}=8.68^{\circ}$ & $\theta_{1}^{*}=16.86^{\circ}$ & $\theta_{1}^{*}=25.31^{\circ}$ & $\theta_{1}^{*}=33.84^{\circ}$ \\
\hline 1 & $\Lambda$ & $\Lambda$ & $\Lambda$ \\
$\theta_{2}^{*}=8.91^{\circ}$ & $\theta_{2}^{*}=17.29^{\circ}$ & $\theta_{2}^{*}=25.43^{\circ}$ & $\theta_{2}^{*}=33.22^{\circ}$ \\
\hline $\mathbf{C}$ & $\mathbf{C}$ & $\mathbf{C}$ & $\mathbf{C}$ \\
$\theta_{3}^{*}=8.93^{\circ}$ & $\theta_{3}^{*}=17.67^{\circ}$ & $\theta_{3}^{*}=25.50^{\circ}$ & $\theta_{3}^{*}=33.29^{\circ}$ \\
\hline
\end{tabular}

(c)

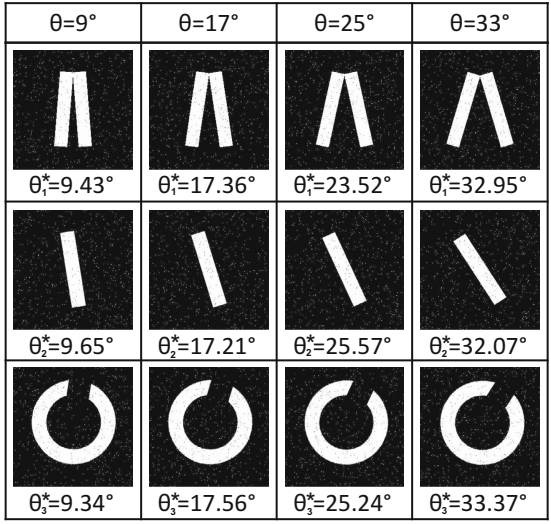

(b)

\begin{tabular}{|c|c|c|c|}
\hline$\theta=9^{\circ}$ & $\theta=17^{\circ}$ & $\theta=25^{\circ}$ & $\theta=33^{\circ}$ \\
\hline$\theta_{1}^{*}=9.12^{\circ}$ & $\theta_{1}^{*}=17.31^{\circ}$ & $\theta_{1}^{*}=24.97^{\circ}$ & $\theta_{1}^{*}=34.49^{\circ}$ \\
\hline$\theta_{2}^{*}=9.27^{\circ}$ & $\theta_{2}^{*}=16.53^{\circ}$ & $\theta_{2}^{*}=23.93^{\circ}$ & $\theta_{2}^{*}=33.27^{\circ}$ \\
\hline$\theta_{3}^{*}=8.74^{\circ}$ & $\theta_{3}^{*}=16.84^{\circ}$ & $\theta_{3}^{*}=25.04^{\circ}$ & $\theta_{3}^{*}=31.97^{\circ}$ \\
\hline
\end{tabular}

(d)

Fig. 3. Some results of test sets: (a) Set 1: free images of degradation. (b) Set 2: images degraded with noise. (c) Set 3: images with scale changed. (d) Set 4: images degraded with noise and with scale changed.

Figure 3 presents some of the results of the test sets. It should be noted that the images shown are not part of the training set. Moreover the effectiveness of the proposed method can be verified using measurements of the estimation error. Table 1 shows the mean squared error (MSE), the mean absolute error (MAE), the mean absolute percentage error (MAPE) and the confidence interval (CI) at $95 \%$ for the different test sets made. 
Table 1. Estimation errors for different test sets made

\begin{tabular}{ccccc}
\hline Test & MSE & MAE & MAPE & CI \\
\hline Set 1 & 0.0085 & 0.0730 & $0.0058 \% \pm 0.1810$ \\
Set 2 & 0.3669 & 0.4946 & $0.0373 \% \pm 1.1873$ \\
Set 3 & 0.3107 & 0.4136 & $0.0343 \% \pm 1.0926$ \\
Set 4 & 0.4110 & 0.5028 & $0.0509 \% \pm 1.2565$ \\
\hline
\end{tabular}

Table 2. Comparative results for estimating the angle of the set of images of Fig. 1(b) with the proposed method and central moments

\begin{tabular}{|c|c|c|c|c|}
\hline & \multicolumn{4}{|c|}{$\bar{\theta}=9^{\circ} \theta=17^{\circ} \theta=25^{\circ} \theta=33^{\circ}$} \\
\hline & $8.95^{\circ}$ & $17.01^{\circ}$ & $24.95^{\circ}$ & $33.06^{\circ}$ \\
\hline Set 1 & $9.00^{\circ}$ & $17.00^{\circ}$ & $25.00^{\circ}$ & $33.00^{\circ}$ \\
\hline osed & $9.65^{\circ}$ & $17.21^{\circ}$ & & $0^{\circ}$ \\
\hline set 2 & $8.26^{\circ}$ & $17.22^{\circ}$ & 25 & \\
\hline Set 3 & $8.91^{\circ}$ & $17.29^{\circ}$ & $25.43^{\circ}$ & $33.22^{\circ}$ \\
\hline set 3 & $9.00^{\circ}$ & $17.00^{\circ}$ & $25.00^{\circ}$ & $33.00^{\circ}$ \\
\hline & $9.27^{\circ}$ & $16.53^{\circ}$ & $23.93^{\circ}$ & $33.27^{\circ}$ \\
\hline Set 4 & $9.34^{\circ}$ & $16.50^{\circ}$ & $24.83^{\circ}$ & $33.66^{\circ}$ \\
\hline
\end{tabular}

The errors presented in Table 1] show an increased error for images with greater degradation. However, for degradation-free images, the proposed method is capable of estimating angles with minimal error even though the images are not part of the training set. Table 2 shows a comparison between the proposed method and the calculation with the central moments for the 4 sets with Fig. 1(b). As can be seen, the method of $\mathrm{Hu}$ with the central moments performs better for sets that were not affected by noise, regardless of changes of scale (sets 1 and 3), and a similar performance to the sets affected by noise (sets 2 and 4). Moreover, computation time for angle estimation is $0.3 \mathrm{~ms}$ in a computer Sony Electronics Inc. Model VAIOß Computer Notebook PC with Intel $\cap$ Core $^{\mathrm{TM}} \mathrm{i} 5-2430 \mathrm{M}$ CPU @ 2.40- $\mathrm{GHz}$ processor, $4.00 \mathrm{~GB}$ of RAM, and the code is implemented using Matlab.

\section{Conclusion}

In conclusion, the proposed method shows how orthogonal moments are used to extract information inherently, such as the angle that is formed on an image from a reference point. Also, the method can adapt to different symmetries as the case of Fig. 1(a), unlike the calculation with the central moments where the principal axes of the method must match with the reference points of the angle. The method of principal axes has better performance for images that rotate in its center and do not have radial symmetry, as in the case of Fig. 1(b). 
Moreover, the proposed recurrence relation facilitates the calculation of Hahn moments. Furthermore, the algorithm has proven to be fast, reliable, and it can be implemented in problems associated with automated visual inspection, among other applications.

\section{References}

1. Mukundan, R., Ong, S.H., Lee, P.A.: Image analysis by Tchebichef moments. IEEE Trans. Imag. Proc. 10, 1357-1364 (2001)

2. Yap, P.T., Paramesran, R., Ong, S.H.: Image analysis by Krawtchouk moments. IEEE Trans. Imag. Proc. 12, 1367-1377 (2003)

3. Yap, P.T., Raveendran, P., Ong, S.H.: Image Analysis Using Hahn Moments. IEEE Trans. Pattern Anal. Mach. Intell. 29, 2057-2062 (2007)

4. Sayyouri, M., Hmimid, A., Qjidaa, H.: Improving the performance of image classification by Hahn moment invariants. J. Opt. Soc. Am. A 30, 2381-2394 (2013)

5. Hu, M.K.: Visual Pattern Recognition by Moment Invariants. IRE Trans. Info. Theory IT-8, 179-187 (1962)

6. Zhu, H., Liu, M., Shu, H., Zhang, H., Luo, L.: General form for obtaining discrete orthogonal moments. IET Image Processing 4, 335-352 (2010)

7. Mukundan, R.: Some computational aspects of discrete orthonormal moments. IEEE Transactions on Image Processing 13, 1055-1059 (2004)

8. Zhang, G., Luo, Z., Fu, B., Li, B., Liao, J., Fan, X., Xi, Z.: A symmetry and birecursive algorithm of accurately computing Krawtchouk moments. Pattern Recognition Letters 31, 548-554 (2010)

9. Zhou, J., Shu, H., Zhu, H.Q., Toumoulin, C., Luo, L.: Image analysis by discrete orthogonal hahn moments. In: Verlag, S. (ed.) International Conference on Image Analysis and Recognition, Toronto, pp. 524-531 (2005)

10. Venkataramana, A., Raj, P.A.: Computation of Hahn Moments for Large Size Images. Journal of Computer Science 6, 1037-1041 (2010)

11. Sheng, Y., Shen, L.: Orthogonal Fourier-Mellin moments for invariant pattern recognition. J. Opt. Soc. Am. A 11, 1748-1757 (1994)

12. Zhu, H., Shu, H., Xia, T., Luo, L., Coatrieux, J.L.: Translation and Scale Invariants of Tchebichef Moments. Pattern Recognition 40, 2530-2542 (2007) 